\title{
BMJ Open Patient and public involvement in health research in low and middle- income countries: a systematic review
}

\author{
Natalie Cook, ${ }^{\oplus 1,2}$ Najma Siddiqi, ${ }^{1,2,3}$ Maureen Twiddy, ${ }^{4,5}$ Richard Kenyon ${ }^{6}$
}

\begin{abstract}
To cite: Cook N, Siddiqi N, Twiddy M, et al. Patient and public involvement in health research in low and middle-income countries: a systematic review. BMJ Open 2019;9:e026514. doi:10.1136/ bmjopen-2018-026514

- Prepublication history and additional material for this paper are available online. To view these files, please visit the journal online (http://dx.doi. org/10.1136/bmjopen-2018026514).
\end{abstract}

Received 25 September 2018 Revised 2 January 2019 Accepted 1 April 2019

Check for updates

(c) Author(s) (or their employer(s)) 2019. Re-use permitted under CC BY-NC. No commercial re-use. See rights and permissions. Published by BMJ.

${ }^{1}$ Department of Health Sciences, University of York, York, UK ${ }^{2}$ Hull York Medical School, York, UK

${ }^{3}$ Bradford District Care NHS Foundation Trust

${ }^{4}$ Institute of Clinical and Applied Health Research, University of Hull, Hull, UK

${ }^{5}$ Leeds Institute of Health

Sciences, University of Leeds, Leeds, UK

${ }^{6}$ Independent Scholar, 0xford, UK

Correspondence to

Dr Natalie Cook;

natalie.cook@york.ac.uk

\begin{abstract}
Objectives Patient and public involvement (PPI) is argued to lead to higher quality health research, which is more relatable to and helps empower the public. We synthesised the evidence to look for examples of PPI in health research in low/middle-income countries (LMICs), looking at levels of involvement and impact. Additionally, we considered the impact of who was undertaking the research on the level of involvement and reported impact.
\end{abstract}

Design Systematic review.

Data sources EMBASE, Medline and PsychINFO, along with hand-searching references, grey literature, Google search and expert advice.

Eligibility criteria Any health research with evidence of patient or public involvement, with no language restrictions dated from 1978 to 1 Dec 2017.

Data extraction and synthesis Data relating to stage and level of involvement, as well as impact, were extracted by one researcher (NC), and a coding framework was developed using an inductive approach to examine the impact of PPI on research. Extracted data were then independently coded by a second lay researcher (RK) to validate the data being collected. Discrepancies were referred to a third independent reviewer (MT) for review and consensus reached.

Results Sixty-two studies met the inclusion criteria. The review revealed the most common stage for PPI was in research planning, and the most common level of involvement was collaboration. Most studies did not provide evidence of effectiveness or elaborate on the impact of PPI, and they tended to report impact from the researcher's perspective. Where impact was mentioned, this generally related to increased relevance to the community, empowerment of participants and alterations in study design.

Conclusions The literature describing approaches to and impact of PPI on LMIC health research is sparse. As PPI is essential to conducting high-quality research, it should be fully reported and evaluated at the end of the research project.

\section{BACKGROUND}

\section{Rationale}

Patient and public involvement (PPI) in research has been defined as 'research being carried out 'with' or 'by' members of the public, rather than 'to', 'about' or 'for' them'. The global mandate for public involvement

\section{Strengths and limitations of this study}

- We conducted an extensive systematic search of the literature, along with hand searching; grey literature searches were limited, however, to a Google search and expert advice due to resource constraints.

- Varying use of terminology relating to patient and public i nvolvement (PPI) over time and across disciplines may mean that some pertinent literature may not have been identified. The literature search was further complicated by the lack of Medical Subject Headings (MeSH) terms in database searches.

- We did not have the resources to seek further information from authors, so data were restricted to published information. Furthermore, the literature search was conducted in December 2017, so recent research may be missed.

- None of the papers identified or reported the impact of PPI from the perspective of the patient or the public, and so any perceived benefits or challenges from these perspectives are missing from the existing literature. Furthermore, papers tend to report PPI within the context of study results, so these often lack details in their reporting.

- Publication and reporting biases might have an impact on the findings of this review, but their impact cannot be estimated.

was set by the World Health Organisation Declaration of Alma-Ata in 1978, as a step towards everyone having the 'right and duty to participate individually and collectively in the planning and implementation of their healthcare'.?

Developing stronger PPI in the research and delivery of healthcare is now a central component of research proposals for funders in high-income countries (HICs), for example, the INVOLVE framework in the UK, which has been well established for over two decades. Patients and the public can be actively involved in research throughout the research cycle, and this can lead to higher quality health research, which meets the needs of the target community, and is relatable to and helps empower the public. 
However, there are concerns that mandating PPI in grant applications can lead to 'tokenistic involvement', with academics involving patients and public in research grants simply for funding purposes, without commitment to embedding them into the research. ${ }^{3}$ The issue of tokenism seems to become even more acute when considering research undertaken in low/middle-income countries (LMICs).

As the emphasis on PPI in research continues to grow in HICs, the extent and impact of PPI in LMICs remains unclear. Research is becoming increasingly globalised, with researchers from HICs operating on an international basis, particularly in LMICs. This research is often supported by smaller local funders, who may not have the same requirements for PPI. HIC-based health research funders expect researchers to engage with the new well-established international, national and institutional sources of guidance about how to undertake research in LMICs, not the least the World Medical Association's Declaration of Helsinki. ${ }^{4}$ This has led to a move to recognise the importance of both economic and cultural differences, and so the importance of identifying locally sustainable solutions.

To our knowledge, there has been one previous attempt to systematically analyse examples of PPI in research in LMICs. Semrau et al conducted a systematic review on service user involvement in mental health system strengthening, concluding that there was no evidence on how best to involve service users in mental health research in LMICs. ${ }^{5}$ In our review, we broadened the search criteria to capture PPI from the whole of health research and also identified examples of PPI, which may be described using a different terminology.

Recently, there has been a growing interest in PPI in LMICs with the launch of the 'International Network for Public Involvement and Engagement in Health and Social Care Research' from Cochrane ${ }^{6}$ and INVOLVE's National Institute for Health Research (NIHR) International Network ${ }^{7}$ to drive PPI forward.

Given this drive to improve PPI in LMICs, it is timely to review the evidence on this important topic. The aim of this systematic review is to describe the PPI strategies and their impact reported in health research in LMICs in a narrative synthesis of the literature.

\section{METHODS}

\section{Eligibility criteria}

Inclusion criteria included

- Any study design.

- Health research.

- Any age of study participants.

- Any language.

- 1978-1 December 2017 (the inception date coincides with WHO Declaration of Alma-Ata).

- Evidence of patient or public involvement in research.

\section{Search strategy and study selection}

A literature search was performed using EMBASE, Medline and PsychINFO, along with hand-searching references of key articles and a Google search and expert advice for grey literature. After consideration, it was decided to include a wide range of terminologies to capture studies that had PPI but did not necessarily define it as such. For example, community-based participatory research (CBPR) and participatory action research (PAR) are commonly used in research, and when the other inclusion criteria were met, these studies were included in the analysis. CBPR is a term used to describe research that ideally stems from the local community and continues to involve all partners, in an equal way, throughout the entire research process, and so closely aligns to 'user-led' research. ${ }^{8}$ Similarly, the term PAR is commonly used to describe an approach that involves 'researchers and participants working together', often focusing on social change. ${ }^{9}$

Studies focusing only on community engagement were excluded unless they specifically included mention of patient or public involvement in the study design or conduct. Community engagement is where 'information and knowledge about research is provided or shared', for example, open meetings to raise awareness or using social media to share findings. ${ }^{10}$ Furthermore, since the study design or topic was not our focus, we included studies of any design from all disciplines; information about PPI may be relevant for health research regardless of study type or discipline.

Search terms were decided with the help of an information specialist (KW), checking for inclusion of key papers known to the team and refining the balance between feasibility and inclusivity. Search terms included

Patient $\mathcal{E}$ Public (patient, public, service-user, care-giver, family, consumer, lay person, advocacy group, NGO, citizen, community, client, consumer, survivor, stakeholder, relative)

AND

Involvement (community participation, patient participation, community based participatory research, PPI, collaborat*, engag*, partner*)

AND

Low and Middle-Income Country (developing country, list of individual countries as per World Bank - Jan 2018)

AND

Health Research (health services research, biomedical research, research design, qualitative)

\section{DATA EXTRACTION AND CODING}

All studies meeting the inclusion criteria were read in full by one researcher (NC), and relevant data, relating to stage and level of involvement as well as impact, were extracted using a structured data extraction sheet and a coding framework was developed using an inductive approach to examine the impact of PPI on research. Extracted data were then independently coded by a second lay researcher (RK) to validate the data being 


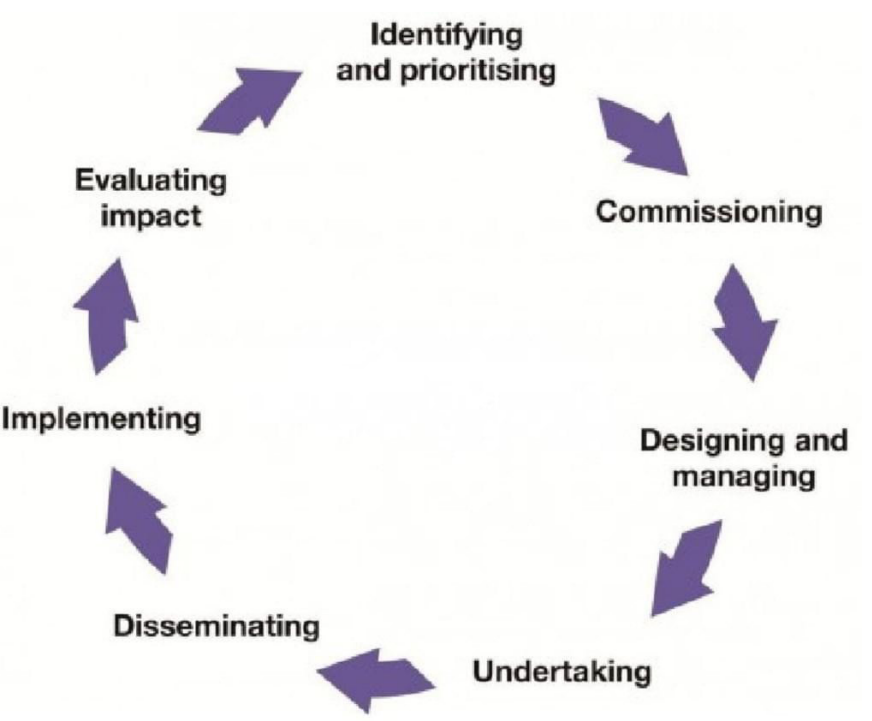

Figure 1 National Institute for Health Research research cycle.

collected. Discrepancies in how the coding framework was applied to extracted data were referred to a third independent reviewer (MT) for review and consensus reached.

In this review, categories for stages of the research cycle were based on those described by the NIHR (see figure 1$)^{11}$ :

For coding purposes, stages were categorised into four groups: pre-research (identifying, prioritising and commissioning); planning (designing and managing); undertaking research (undertaking); postresearch (disseminating, implementing and evaluating impact). Levels of involvement were coded using NIHR definitions ${ }^{12}$ :

1. Consultation, which is asking the public for their views and using them to inform decision making.

2. Collaboration, which consists of an 'ongoing partnership' between research teams and members of the public, 'where decisions about the research are shared'.

3. User-controlled research, which is 'actively controlled, directed and managed by' the public.

No quality assessment was performed on the studies, since the purpose of the review was to identify strategies and the impact of PPI rather than focus on the type or the quality of the study undertaken. However, in order to assist with the interpretation of the results, we extracted key information relating to research design (see online appendix 1).

Finally, as part of the review, we designed and led a workshop attended by LMIC partners from the Improving Mental and Physical Health Multimorbidity and Developing Research Capacity (IMPACT) Group on 7 December 2018 in York. In this, we presented our findings and led a group discussion covering PPI terminology, recruitment strategies and ways of reporting impact, all of which will feed into the IMPACT study design.
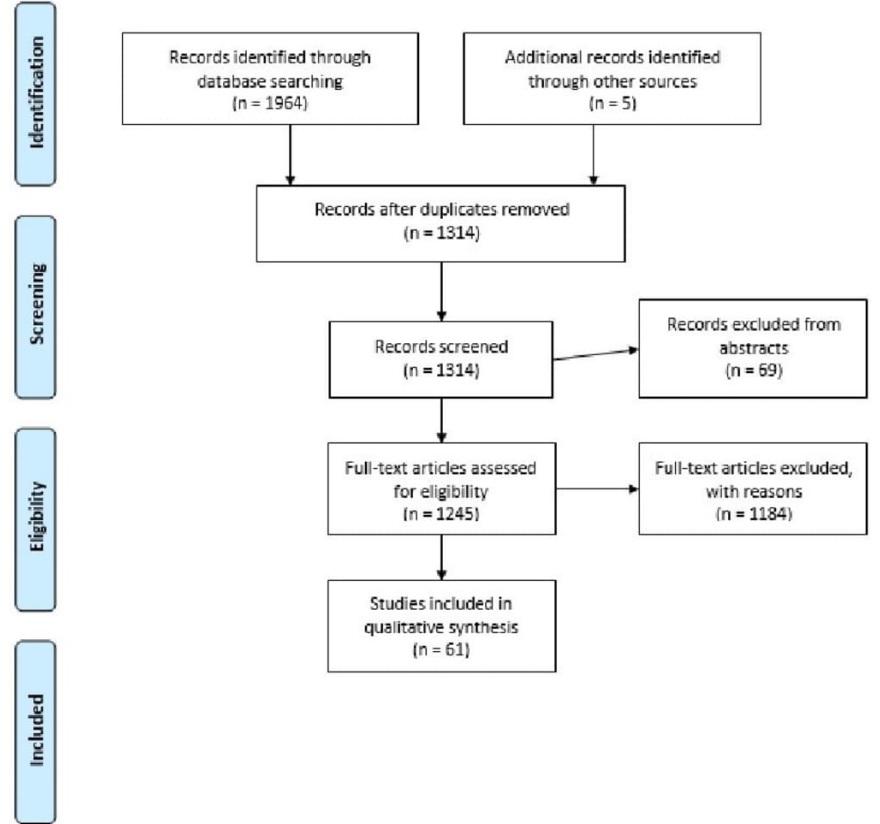

Figure 2 Preferred Reporting Items for Systematic Reviews and Meta-Analyses flow diagram.

\section{PATIENT AND PUBLIC INVOLVEMENT}

This narrative review was supported by RK, who is a lay researcher who assisted as an independent coder and author. Additionally, LMIC partners from IMPACT reviewed the findings and contributed to the discussion, as described above.

\section{RESULTS}

A total of 1969 studies were identified in the literature search (see figure 2 for Preferred Reporting Items for Systematic Reviews and Meta-Analyses (PRISMA) flow diagram). After duplicate removal, 1314 abstracts (and full papers, if required) were screened based on the inclusion criteria by one researcher (NC), resulting in 1184 studies being excluded (see figure 3 for details of exclusion criteria). A total of 61 studies from 34 different countries were included in the narrative synthesis (see online appendix 1 and 2).

Many of the included studies identified their method as CBPR $(n=26)$ or PAR $(n=11)$. The majority of the papers were primary research; either developing or piloting interventions $(n=20)$, qualitative research $(n=17)$, research methods/design $(n=6)$ or research priorities $(n=4)$. In these papers, discussion of PPI was generally secondary to reporting the study results and often comprised only a few sentences scattered throughout the paper. The remaining papers were reflections on research $(\mathrm{n}=14)$, particularly focused on community advisory boards and researcher experiences. None of the studies identified would meet the criteria of the only agreed reporting framework for PPI, the Guidance for Reporting Involvement of Patients and the Public (GRIPP) 2 checklist. ${ }^{13}$ 


\begin{tabular}{|l|l|}
\hline Reason & $\begin{array}{l}\text { Number of } \\
\text { studies }\end{array}$ \\
\hline Not a LMIC & 263 \\
\hline Not health research & 247 \\
\hline No PPI & 358 \\
\hline PPI Theory & 157 \\
\hline Ethics Research & 101 \\
\hline Patient/Community Engagement & 46 \\
\hline Service Development & 10 \\
\hline Not in English & 1 \\
\hline $\begin{array}{l}\text { Duplicate (not picked up in initial duplicate } \\
\text { removal) }\end{array}$ & 1 \\
\hline \multicolumn{2}{|l|}{ Total = 1184 } \\
\hline
\end{tabular}

Figure 3 Exclusion criteria. LMIC, low/middle-income country; PPI, patient and public involvement.

The review found that the most common stage to have PPI was the planning stage $(\mathrm{n}=51)$ followed by undertaking $(n=30)$, postresearch $(n=27)$ and, finally, pre-research $(\mathrm{n}=18)$. Regarding level of involvement, 37 studies were classed as collaboration, with only 4 being classed as user controlled and 20 as consultation (see online appendix 1). Most studies took place in the African subcontinent, followed by India. Using World Bank criteria, the countries where the research took place could be classified as low-income countries $(n=8)$, lower middle-income countries $(\mathrm{n}=15)$ and upper middle-income countries $(\mathrm{n}=11)^{14}$ (see online appendix 2).

Studies with consultation-level involvement often had some form of community advisory committee that was used to inform the community about different aspects of the research and sometimes seek their opinion on the research objectives, design and implementation. Mushi et al describe presenting preliminary findings and intervention packages in village meetings as part of the consultation process. ${ }^{15}$ Similarly, Owolabi et al reports using a task force that included representatives of the Nigerian Stroke Society to 'review the progress of the community participatory research process and make recommendations about any local adaptations to facilitate its effectiveness'. ${ }^{16}$

Collaboration-level involvement had varying strategies; some studies described a partnership between researcher and advocacy groups that ensured ongoing, two-way engagement between the community and the research team, with the community identifying the healthcare problem and driving forward solutions. Bradley and Puane research started after the community voiced concerns to community health workers (CHWs) about the increasing prevalence of hypertension and diabetes in their community. ${ }^{17}$ Following this, a meeting was held with the local community health committee and community leaders. Aims were formulated with the intention of engaging CHWs in many aspects of the research process, including data collection, analysis and dissemination. Zola et al reported that the "community based organisation members (CBO), people living with HIV (PLHIV) and researchers were involved, in an equitable partnership'. ${ }^{18}$ Community members were trained in research methods and ethics, and then involved in developing the questionnaire and conducting the interviews. ${ }^{18}$

Finally, looking at user-controlled research, common strategies included community-initiated research, involvement in the entire research project, from pre-research through to evaluation and ongoing involvement from peer researchers. ${ }^{19-22}$ Hayashi et al describe CBPR, which was led by a group of active and former drug users (Thai Drug Users' Network) who were involved in the whole study from design to analysis and dissemination. ${ }^{22}$ Similarly, Jongudomkarn's study was initiated by community members via a forum, and the women were involved in the entire research process right through to the action plan and evaluation. ${ }^{21}$

The most commonly cited impacts of PPI included increased relevance to the community, empowerment of participants and alterations in trial design. Foster et al reported that the research team, consisting of US nurses, Dominican nurses and community leaders, continued to meet after the research had ended to drive improvement, hence empowering the team. ${ }^{23}$ After the conclusion of Jongudomkarn's research, the women involved became the "resource persons responsible for alcohol consumption campaigns'. ${ }^{21}$ Liu et al explained that the community steering group modified the wording of some of the translations to ensure cultural relevance for a Mandarin-speaking population. ${ }^{24}$ Following feedback from the community reference team, Mosavel et al decided to 'refocus the research from cervical cancer to "cervical health"'. ${ }^{25}$

Another benefit reported was improved quality of results; Bowling etal commented that the 'partnership with local researchers and community partners strengthened the quality of the findings through their involvement in design, recruitment and interpretation phases' ${ }^{26}$ Reflecting on higher levels of engagement in the community, Grinker etal described how PPI 'facilitates the crucial recruitment phase as well as participant retention by limiting or managing negative views or misunderstandings of the researchers, procedures or goals of the study ${ }^{27}$ Another impact to consider is increased community trust and improved community-researcher relations. In Simwinga etal 's South African study, community advisory board members provided a 'protective role for community members' and also helped 'resolve tensions between researchers and community'. ${ }^{28}$ Furthermore, PPI can help challenge common community misconceptions and stigma; Adhikari et al noted that having local villagers involved in their malaria study helped tackle rumours 
in the community. ${ }^{29}$ Finally, some studies reported on the difficulties of PPI, largely focusing on the extra time and money required to have PPI in their study. ${ }^{22}{ }^{30-32}$ It is important to note that most of the impact reported is from the researcher's perspective and was often reported as an aim rather than an evidence-based outcome of PPI. In some cases, impact was not reported at all.

We thought it was important to consider whether the study authors were from the study country. The vast majority of studies $(93 \%, \mathrm{n}=57)$ had at least one author from the study country; 43 of these were in partnership with researchers from HICs. Only five studies were conducted without the input of local researchers. Although it is difficult to be certain from study reports, we estimate 10 of the 61 studies included non-governmental organisation or advocacy group members as authors.

Finally, we struggled to find funding guidance to identify whether PPI was a required component of the research. Some of the larger international funders mention public engagement as a concept in their material. ${ }^{33-36}$ However, many of the studies were funded by smaller local funders, for whom we were unable to find guidance.

\section{DISCUSSION}

This review is the first to systematically review PPI in health research in LMICs. None of the studies made explicit reference to PPI as a term nor did they refer to the use of any tools or funding requirements. This could reflect an actual lack of PPI but may also reflect that research teams are simply not reporting PPI in research publications, or that researchers are using a different terminology for involvement activities. It is important to state that the poor reporting of PPI is not unique to research conducted in LMICs. Both Mockford et al and Brett have identified issues with the evidence base behind PPI, as well as the poor quality of reporting, which is essentially limiting our understanding of PPI impact on HICs. ${ }^{37} 38$

Nevertheless, the findings reveal that researchers are using PPI at different stages and levels in health research taking place in LMICs, highlighting that regardless of the subject, type or location of research, PPI can be integrated into the research process and may consequently have an impact on both the research and the individuals involved. There is a lack of reporting of PPI strategies and impact; those studies that do are still largely reporting impact from the researcher's perspective.

Earlier research looking at the impact of PPI in the USA, UK and Europe aligns closely with the findings of our review of LMICs, suggesting that although the context may be quite different, benefits can be realised from PPI in LMIC. Brett et al discuss the impact of PPI throughout the research cycle, suggesting that PPI in the planning stage can help identify and prioritise topics according to relevance; in the implementation stage, it can help participant recruitment and researcher-participant rapport, and during analysis and write-up, it allows findings to be interpreted from a user perspective and can also assist with research dissemination. ${ }^{39}$

Our review identified that most PPIs in LMICs take place during the planning stage of research, which is in contrast to a similar review, not focused on LMIC, which found more examples of PPI in the execution phase. ${ }^{40}$ It is difficult to say definitively why this might be the case, but it may be related to non-LMIC researchers recognising the need to gain local knowledge in planning research in relatively unfamiliar settings.

PPI for many studies involved the setting up of community advisory groups/boards, but it is not clear if this is the most appropriate source of PPI or whether the formation of these groups acts as a barrier to meaningful engagement with the end user of the research, as the members of these groups were local community leaders, rather than those living with the particular health problem.

Furthermore, the involvement of local researchers was also apparent in the studies identified. This provided research teams with a mechanism to liaise at a grass roots level with local leaders in their local dialects and to remain alert to local sensitivities. In addition, LMIC researchers may gain an advantage by identifying topics relevant to the community and by gaining access and acceptance into the community, ultimately enabling the progression of the research project; this could also be used as a useful opportunity for research capacity building, which was mentioned in a couple of included studies. ${ }^{22} 41$ The overall relatively low level of patient involvement across the research cycle may be due to the importance, or lack of it, that funders place on PPI. Many of the studies reported in our review were supported by universities or smaller funders, for whom we were unable to find funding guidance. Larger, national and international funders do provide information about engaging communities but tend not to use the term 'patient involvement'; rather they focus more on public engagement, which, as a concept, has some overlap with community engagement and PPI. For example, National Institutes of Health, USA, encourages community engagement as a 'process of working collaboratively'. ${ }^{34}$ Similarly, guidance issued by the Australian Government Research Award Scheme emphasises the importance of 'research engagement and communication', 35 and the UNC Centre for AIDS Research has a 'Strategic Community Engagement Education Dissemination Office ${ }^{36}$ with similar aims.

It is therefore unsurprising that researchers focus on the engagement of community leaders as this aligns with those goals, rather than engaging with research participants. What is unclear in the literature from LMICs is whether these were the goals of PPI in these studies, or incidental benefits. The rationale given by many of these researchers for involving people at the design stage is often related to ensuring that gatekeepers support the research and facilitate access to the population of interest, rather than to improve the quality of the research per se. ${ }^{42-44}$ Many of the studies included in the review describe research in populations that would be 
defined as hard-to-reach groups, such as people with or at risk of HIV/AIDS, people with mental health problems (eg, schizophrenia) and people with drug/alcohol problems. In the UK, there is now a long-established literature setting out both consumerist and democratic reasons for involving patients and the public in research. ${ }^{45}$ It could be argued that in at least some of the studies, we found that a third reason dominated, and that was pragmatism.

Considering ways of increasing potential for impact, Brett $e t$ al reflected that good training and having clearly defined roles, in a positive supportive environment with mutual trust and respect would be beneficial. ${ }^{39}$ Similarly, the EPIC study encourages researchers to set clear goals and well-developed plans and to advocate for individuals to be involved early in the research in a responsive, managerial way rather than solely to have a general oversight, more often seen in membership of a steering committee (the latter approach was widely used in the studies we identified in our review) ${ }^{46}$ Since many of the studies included in our review were CBPR, there was often a more hands-on type of involvement from participants, for example, trained community members taking on the role of researcher ${ }^{47-49}$ and participants shaping/ piloting interventions ${ }^{5051}$; this type of involvement will also fulfil the secondary aim of engaging participants in the research and perhaps develop local research capacity.

Finally, lack of standardisation in designing and evaluating PPI frameworks and strategies means it is difficult for researchers to develop a comprehensive PPI strategy. Over the last decade, researchers have been developing PPI toolkits but as yet, none have been adopted as a standardised tool. ${ }^{52-54}$ One of the more recent is the GRIPP2 checklist, which was developed following a systematic review and Delphi study to assist with the reporting of PPI in research, with the aim of improving quality and transparency of the PPI evidence base. ${ }^{13}$ The authors recommend that the checklist should be used prospectively in research design and retrospectively in evaluation. The GRIPP2 short form includes sections on aims, method, results (both positive and negative), discussion (impact) and reflection, each of these areas requiring information specific to PPI. ${ }^{13}$ However, it is important to note that this tool, though developed for international use, was developed from an HIC perspective, and though there are similarities, there are also complexities specific to LMIC that need to be considered, particularly the variations in research infrastructure, cultural differences, the power differential between researcher and researched in these contexts, and often, lower research budget. Other than cultural differences and research budget, none of the other areas were explicitly considered in the included studies.

The review suggests that there are positive gains to be had from involving communities from LMICs in research, and the complexities faced by LMIC research are things that PPI can help with, through facilitating communication with communities ${ }^{28} 55$ and adapting interventions for different cultures. ${ }^{50} 56$ PPI is still relatively new, even in countries with a well-established research tradition; it may take time for it to gain traction in countries without this tradition.

\section{CONCLUSION}

From this study, we can conclude that PPI does happen in LMIC health research but is generally described using a different terminology and rarely are detailed PPI strategies published. Similarly, at present the impact of PPI on both the participant and research is poorly documented. There is significant work needed to encourage closer engagement with end users of research, not just with community 'gate-keepers'.

To improve this, LMIC research funders and journal publishers should make PPI an explicit requirement. Work also needs to be done on adapting pre-existing PPI tools for use in LMIC and encouraging their use, to clearly evidence the level, stage and impact of PPI. This will give researchers a generic format and space for reflection and will also capture the voice of the patient and the public to show how it has affected them as individuals, as well as the wider community.

Acknowledgements We would like to thank Kath Wright for her support with the literature search and Joseph Clarke for his feedback on the manuscript. We would also like to acknowledge the contribution of LMIC partners from the Improving Mental and Physical Health Multimorbidity and Developing Research Capacity (IMPACT) Group, including Dr Aliya Naheed, International Centre for Diarrhoeal Disease Research, Dhaka, Bangladesh; Professor Rumana Huque, ARK Foundation, Dhaka, Bangladesh; Dr Faruq Alam, National Institute of Mental Health, Dhaka, Bangladesh; Professor AHM Enayet Hussain, Ministry of Health and Family Welfare, Bangladesh; Professor Santosh Chaturvedi, National Institute of Mental Health and Neurosciences, Bangalore, India; Professor Pratima Murthy, National Institute of Mental Health and Neurosciences, Bangalore, India; Dr Arun Kandasamy, National Institute of Mental Health and Neurosciences, Bangalore, India; Dr Krishna Prasad, National Institute of Mental Health and Neurosciences, Bangalore, India; Profesor Asad Nizami, Institute of Psychiatry, Rawalpindi, Pakistan; and Dr Faiza Aslam, Institute of Psychiatry, Rawalpindi, Pakistan.

Contributors NC, NS and MT designed the study. NC extracted, analysed, synthesised and coded the data. MT and RK independently coded the data. NC wrote the first draft of the manuscript. NS, MT and RK contributed to the interpretation of the results and the writing of the manuscript for publication.

Funding This research was led by a National Institute for Health Research (NIHR) Academic Clinical Fellow. The study was part of a programme of research (Improving Mental and Physical Health Multimorbidity and Developing Research Capacity (IMPACT)) that is commissioned by the NIHR using Official Development Assistance funding (grant GHR 17/63/130). The views expressed in this publication are those of the authors and are not necessarily those of the National Health Service, the NIHR or the Department of Health and Social Care.

Competing interests None declared.

Patient consent for publication Not required.

Provenance and peer review Not commissioned; externally peer reviewed.

Data sharing statement Studies included in the research are found in appendix 1.

Open access This is an open access article distributed in accordance with the Creative Commons Attribution Non Commercial (CC BY-NC 4.0) license, which permits others to distribute, remix, adapt, build upon this work non-commercially, and license their derivative works on different terms, provided the original work is properly cited, appropriate credit is given, any changes made indicated, and the use is non-commercial. See: http:// creativecommons.org/licenses/by-nc/4.0/. 


\section{REFERENCES}

1. INVOLVE. Frequently asked questions. 2018 http://www.invo.org.uk/ frequently-asked-questions/ (Accessed 12 Jun 2018).

2. World Health Organisation. Declaration of Alma Ata: report of the International Conference on Primary Health Care. Geneva, 1978.

3. Lemonsky F. Service user involvement in research. Lancet Psychiatry 2015;2:780.

4. World Medical Association. Declaration of Helsinki - Ethical Principles for Medical Research involving Human Subjects. 2008.

5. Semrau M, Lempp H, Keynejad R, et al. Service user and caregiver involvement in mental health system strengthening in low- and middle-income countries: systematic review. BMC Health Serv Res 2016;16:79.

6. Cochrane. An international network for public involvement and engagement in health and social care research. http://www. cochrane.org/news/international-network-public-involvement-andengagement-health-and-social-care-research (Accessed 12 Jun 2018)

7. INVOLVE (no date). International Working. http://www.invo.org.uk/ current-work/international-working/.

8. Minkler M. Community-based research partnerships: challenges and opportunities. J Urban Health 2005;82:ii3-ii12.

9. Participatory Methods. Participatory Action Research. http://www. participatorymethods.org/glossary/participatory-action-research (Accessed 3 Dec 2018).

10. National Institute of Health Research. Patient, Carer and Public Information. https://www.nihr.ac.uk/nihr-in-your-area/kent-surreyand-sussex/patient-and-public-involvement-and-engagement.htm (Accessed 3 Dec 2018).

11. National Institute of Health Research. The Research Cycle. https:// www.nihr.ac.uk/patients-and-public/how-to-join-in/the-researchcycle/ (Accessed 12 Jun 2018).

12. National Institute of Health Research. Approaches to public involvement in research. http://www.invo.org.uk/posttyperesource/ approaches-to-public-involvement/ (Accessed 12 Jun 2018).

13. Staniszewska S. GRIPP2 reporting checklists: tools to improve reporting of patient and public involvement in research. British Medical Journal 2017:358.

14. World Bank. World Bank Country and Lending Groups. 2018 https:// datahelpdesk.worldbank.org/knowledgebase/articles/906519 (Accessed 22 Dec 2018).

15. Mushi D, Mpembeni R, Jahn A. Effectiveness of community based Safe Motherhood promoters in improving the utilization of obstetric care. The case of Mtwara Rural District in Tanzania. BMC Pregnancy Childbirth 2010;10:14.

16. Owolabi MO, Akinyemi RO, Hurst S, et al. Tailored Hospital-based Risk Reduction to Impede Vascular Events After Stroke (THRIVES) study: qualitative phase protocol. Crit Pathw Cardiol 2014;13:29-35

17. Bradley HA, Puoane T. Prevention of hypertension and diabetes in an urban setting in South Africa: participatory action research with community health workers. Ethn Dis 2007;17:49-54.

18. Zola EK, Gifudu GM, Henry E, et al. Factors associated with HIV voluntary disclosure of people living with HIV to their steady sexual partner in the Democratic Republic of the Congo: results from a community-based participatory research. Pan Afr Med J 2014;19.

19. Aitaoto N, Campo S, Snetselaar LG, et al. Formative research to inform nutrition interventions in Chuuk and the US Pacific. J Acad Nutr Diet 2015;115:947-53.

20. Hann K, Pearson H, Campbell D, et al. Factors for success in mental health advocacy. Glob Health Action 2015;8:28791.

21. Jongudomkarn D. A volunteer alcohol consumption reduction campaign: participatory action research among Thai women in the Isaan region. Asian Pac J Cancer Prev 2014;15:7343-50.

22. Hayashi K, Fairbairn N, Suwannawong $P$, et al. Collective empowerment while creating knowledge: a description of a community-based participatory research project with drug users in Bangkok, Thailand. Subst Use Misuse 2012;47:502-10.

23. Foster J, Burgos R, Tejada C, et al. A community-based participatory research approach to explore community perceptions of the quality of maternal-newborn health services in the Dominican Republic. Midwifery 2010;26:504-11.

24. Liu J, McCauley L, Leung P, et al. Community-based participatory research (CBPR) approach to study children's health in China: experiences and reflections. Int J Nurs Stud 2011;48:904-13.

25. Mosavel M, Simon C, van Stade D, et al. Community-based participatory research (CBPR) in South Africa: engaging multiple constituents to shape the research question. Soc Sci Med 2005;61:2577-87.

26. Bowling J, Dodge B, Banik S, et al. Perceived health concerns among sexual minority women in Mumbai, India: an exploratory qualitative study. Cult Health Sex 2016;18:826-40.
27. Grinker RR, et al. "Communities" in community engagement: lessons learned from Autism Research in South Korea and South Africa. Autism Research 2012;5:201-10.

28. Simwinga M, Bond V, Makola N, et al. Implementing Community Engagement for Combination Prevention: Lessons Learnt From the First Year of the HPTN 071 (PopART) Community-Randomized Study. Curr HIVIAIDS Rep 2016;13:194-201.

29. Adhikari B, Phommasone K, Pongvongsa T, et al. Factors associated with population coverage of targeted malaria elimination (TME) in southern Savannakhet Province, Lao PDR. Malar J 2017;16:424.

30. Fujiwara T, Takano T, Nakamura K, et al. The spread of drug abuse in rapidly urbanizing communities in Vientiane, Lao People's Democratic Republic. Health Promot Int 2005;20:61-8.

31. Karmaliani R, McFarlane J, Asad N, et al. Applying community-based participatory research methods to improve maternal and child health in Karachi, Pakistan. Nurs Outlook 2009;57:204-9.

32. Kobeissi L, Nakkash R, Ghantous Z, et al. Evaluating a community based participatory approach to research with disadvantaged women in the southern suburbs of Beirut. $J$ Community Health 2011;36:741-7.

33. Welcome Trust (no date. Planning your public engagement. https:// wellcome.ac.uk/funding/guidance/planning-your-public-engagement (Accessed 3 Dec 2018).

34. National Institutes of Health (NIH). Community Engagement. https:// www.nih.gov/health-information/nih-clinical-research-trials-you/ community-engagement (Accessed 3 Dec 2018).

35. Australian Government. Department of Foreign Affairs and Trade. ADRAS Guidelines. 2012 https://dfat.gov.au/about-us/ publications/Documents/adras-guidelines-2012.pdf.

36. UNC Center for AIDS Research (UNCCAF). Strategic Community Engagement Education Dissemination (SCEED) Office. http:// unccfar.org/portfolio/strategic-community-engagement-educationdissemination-sceed-office/ (Accessed 3 Dec 2018)

37. Mockford C, Staniszewska S, Griffiths F, et al. The impact of patient and public involvement on UK NHS health care: a systematic review. Int J Qual Health Care 2012;24:28-38.

38. Brett J. The PIRICOM Study: a systematic review of the conceptualisation, measurement, impact and outcomes of Patients and Public Involvement in Health and Social Care Research. Warwick: University of Warwick, 2010.

39. Brett J, Staniszewska S, Mockford C, et al. Mapping the impact of patient and public involvement on health and social care research: a systematic review. Health Expect 2014;17:637-50.

40. Domecq JP, Prutsky G, Elraiyah T, et al. Patient engagement in research: a systematic review. BMC Health Serv Res 2014;14:89.

41. Maman S, Lane T, Ntogwisangu J, et al. Using participatory mapping to inform a community-randomized trial of HIV counseling and testing. Field methods 2009;21:368-87.

42. Campbell MM, Susser E, de Vries J, et al. Exploring researchers' experiences of working with a researcher-driven, populationspecific community advisory board in a South African schizophrenia genomics study. BMC Med Ethics 2015;16:45.

43. Ntshanga SP, Ngcobo PS, Mabaso ML, et al. Establishment of a Community Advisory Board (CAB) for tuberculosis control and research in the Inanda, Ntuzuma and KwaMashu (INK) area of KwaZulu-Natal, South Africa. Health Policy 2010;95(2-3):211-5.

44. Remien $\mathrm{RH}$, Mellins $\mathrm{CA}$, Robbins RN, et al. Masivukeni: development of a multimedia based antiretroviral therapy adherence intervention for counselors and patients in South Africa. AIDS Behav 2013:17:1979-91.

45. Beresford P. User Involvement in Research and Evaluation: Liberation or Regulation? Social Policy and Society 2002;1:95-105.

46. Gamble C. An evidence base to optomise methods for involving patient and public contributors in clinical trials: a mixed methods study. Southampton: Health Service and Delivery Research (339) NIHR Journals Library, 2015.

47. Lorway R, Thompson LH, Lazarus L, et al. Going beyond the clinic: confronting stigma and discrimination among men who have sex with men in Mysore through community-based participatory research. Crit Public Health 2014;24:73-87.

48. Morisky DE, Malow RM, Tiglao TV, et al. Reducing sexual risk among Filipina female bar workers: effects of a CBPRdeveloped structural and network intervention. AIDS Educ Prev 2010;22:371-85

49. Nichter M, Padmajam S, Nichter M, et al. Developing a smoke free homes initiative in Kerala, India. BMC Public Health 2015;15:480.

50. Baptiste DR, Bhana A, Petersen I, et al. Community collaborative youth-focused HIV/AIDS prevention in South Africa and Trinidad: preliminary findings. J Pediatr Psychol 2006;31:905-16.

51. Blanchard AK, Sangha CA, Nair SG, et al. Pursuing authenticity from process to outcome in a community-based participatory research 
study of intimate partner violence and HIV Vulnerability in North Karnataka, India. Qual Health Res 2017;27:204-14.

52. Bagley HJ, Short H, Harman NL, et al. A patient and public involvement (PPI) toolkit for meaningful and flexible involvement in clinical trials - a work in progress. Res Involv Engagem 2016;2:15.

53. Cartwright J, Crowe S. Patient and Public Involvement Toolkit. BMJ Books 2011.

54. Scottish Health Council. The Participation Toolkit. 2018 http://www. scottishhealthcouncil.org/patient_public_participation/participation_
toolkit/the_participation_toolkit.aspx\#.VZvZybFwblU (Accessed 12 Jun 2018).

55. Shanks L, Moroni C, Rivera IC, et al. "Losing the tombola": a case study describing the use of community consultation in designing the study protocol for a randomised controlled trial of a mental health intervention in two conflict-affected regions. BMC Med Ethics 2015;16:38.

56. Pardo G, Saisaengjan C, Gopalan P, et al. Cultural adaptation of an evidence-informed psychosocial intervention to address the needs of PHIV+ Youth in Thailand. Global Social Welfare 2017;4:209-18. 